Reprinted from:

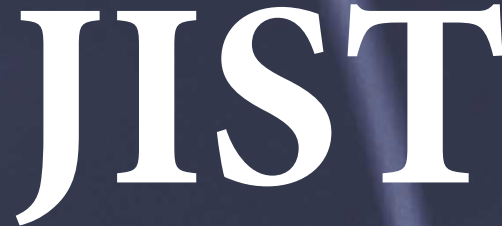

Vol. 53, No. 5 September/October 2009
Journal of

Imaging Science

\section{and Technology}

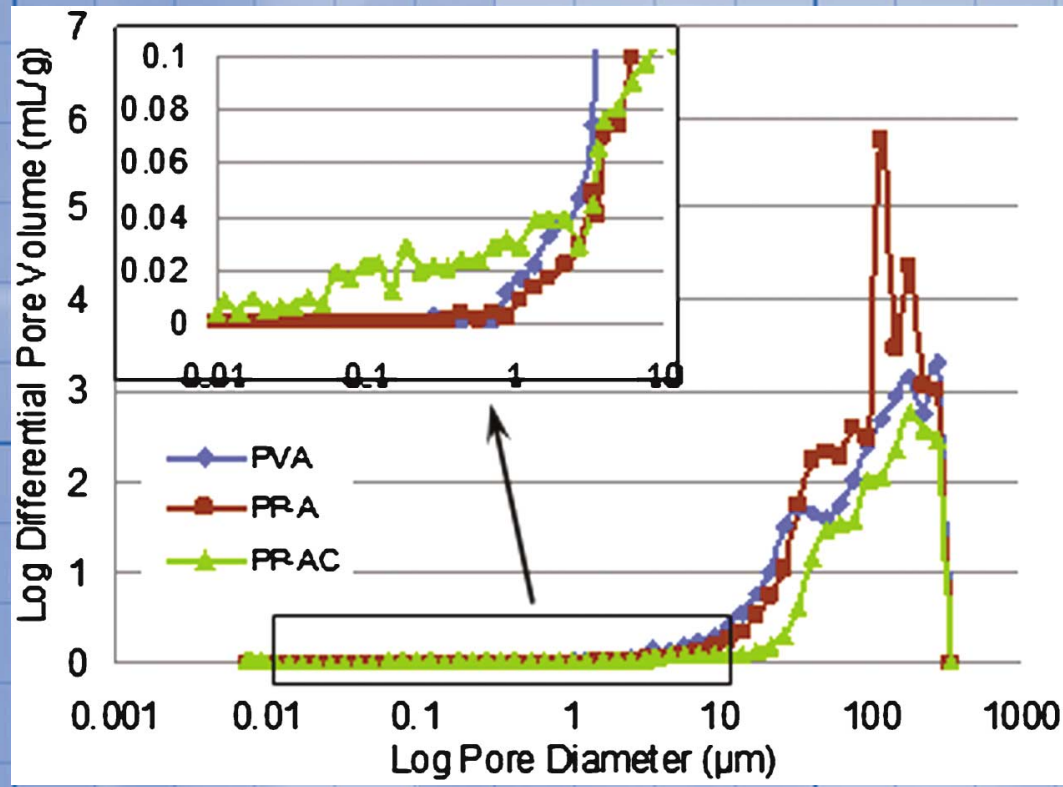

Society for Imaging Science and Technology 



\title{
Electronic Spectra of the 1:1 Rhodamine B Base with Ethyl Gallate in Solution and in the Solid State
}

\author{
K. Sato, H. Shima and J. Mizuguchi ${ }^{-}$ \\ Graduate School of Engineering, Yokohama National University, 240-8501 Yokohama, Japan \\ E-mail: mizu-j@ynu.ac.jp
}

\begin{abstract}
The electronic spectra of the 1:1 rhodamine $B$ base (RBB: leuco dye) with ethyl gallate (EG: developer) have been studied in solution and in the solid state (i.e., in spin-coated films and in single crystals) on the basis of the crystal structure analysis. There are two crystalline phases in the 1:1 "RBB/EG" colorant at low (93 K) and room temperatures. In solution, the maximum color intensity occurs with the 1:1 molar ratio of $\mathrm{RBB}$ with $\mathrm{HCl}$, giving an absorption band at about $556 \mathrm{~nm}$. In the solid state of spin-coated RBB/EG layers, an absorption band appears around $577 \mathrm{~nm}$ due to the ring opening caused by the hydrogen bond formation between RBB and EG. However, the color intensity is found to be limited to about $80 \%$ of the maximum available value. This has been attributed to a residual fraction of RBB molecules whose lactone ring is still closed due to steric hindrance. In addition, the polarized reflection spectra measured on single crystals of RBB/EG exhibit a drastically different spectrum (i.e., absorption maximum about $480 \mathrm{~nm}$ ) from that of spin-coated films of the amorphous state. This result suggests that strong excitonic interactions of the $\mathrm{H}$-aggregate type are operative in single crystals of RBB/EG that significantly displace the absorption band toward shorter wavelengths. () 2009 Society for Imaging Science and Technology.

[DOI: 10.2352/J.ImagingSci.Technol.2009.53.5.050303]
\end{abstract}

\section{INTRODUCTION}

Leuco dyes are known to form brilliant colors when their lactone rings are opened, either by protonation in solution or by hydrogen bond formation in the solid state. ${ }^{1-10}$ Therefore, leuco dyestuffs are widely used in practice for facsimile papers as well as for rewritable media., ${ }^{2,10}$ One of the typical examples of the leuco system is shown in Figure 1(a), where rhodamine $\mathrm{B}$ base (RBB) is a leuco dye and ethyl gallate (EG) is a phenolic acid used as a developer. ${ }^{10}$ The coloration is considered to arise from the opening of the lactone ring, leading to the formation of a push-pull system composed of the donor site $\left(-\mathrm{N}\left(\mathrm{CH}_{3}\right)_{2}\right)$ and the acceptor site $\left(=\mathrm{N}^{+}\left(\mathrm{CH}_{3}\right)_{2}\right)$ in the xanthene ring as shown in Fig. 1(b). However, the driving force of the opening of the lactone ring is different in solution from that in the solid state. In solution, the proton of an acid, for example, hydrochloric acid, opens the lactone ring (-O-) to form a carboxyl group $(-\mathrm{COOH})$ [Figure 2(a)]. On the other hand, in the solid state, hydrogen bond formation between the carboxylate $\left(-\mathrm{COO}^{-}\right)$of $\mathrm{RBB}$ and the $\mathrm{OH}$ groups of EG induces the ring

\section{IS\&T Member.}

Received Dec. 22, 2008; accepted for publication May 19, 2009; published online Aug. 27, 2009.

$1062-3701 / 2009 / 53(5) / 050303 / 7 / \$ 20.00$. opening [Fig. 2(b)]. The opening of the lactone ring (i.e., hydrogen bond formation) and its closure (i.e., collapse of the hydrogen bond) by heating are utilized in practice for the reversible imaging systems employing leuco dyestuffs. ${ }^{2,3,10}$

As described above, the color generation mechanism is simply the opening of the lactone ring. However, the extent of the ring opening exerts a profound influence on the absorption maximum in the spectral region as well as on its tinctorial strength. Especially, in the solid state, spectral displacement takes place quite often in colorant systems (i.e., dyes and pigments) due to intermolecular interactions, socalled excitonic interaction. ${ }^{11,12}$ This interaction is quite significant in molecular crystals where the molecules with a high absorption coefficient are periodically arranged. These unexplored questions motivated us to study the crystal and electronic structures of the RBB/EG system with special attention to the tinctorial strength and spectral changes in solution and in the solid state.

The structures of leuco colorants of both the triphenyl methane and fluoran types were reported by Rihs and Weis. ${ }^{13,14}$ On the other hand, the structure of RBB/EG at room temperature was studied by Sekiguchi et al. ${ }^{10}$ The $\mathrm{RBB} / \mathrm{EG}$ complex forms a dimer (RBB $\cdots \mathrm{EG} \cdots \mathrm{EG} \cdots \mathrm{RBB})$<smiles>CCN(CC)c1ccc2c(c1)Oc1cc(N(CC)CC)ccc1C21OC(=O)c2ccccc21</smiles>

RBB

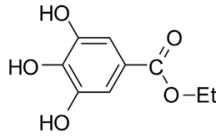

EG (a)<smiles>CCN(CC)c1ccc2c(C)c3ccc(=[N+](CC)CC)cc-3oc2c1</smiles>

(Acceptor) Chromophore (Donor)

(b)

Figure 1. (a) Molecular conformation of RBB (colorant) and EG (developer) and (b) the typical push-pull system of the xanthene moiety due to the opening of the lactone ring, consisting of the chromophore and the auxochromes. 
(a)

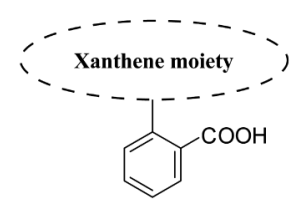

in solution (b)

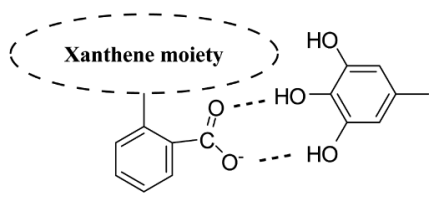

in the solid state
Figure 2. (a) lactone ring opening due to protonation in solution and (b) due to hydrogen bond formation in the solid state.

through intermolecular $\mathrm{OH}^{\cdots} \mathrm{O}$ hydrogen bonds. In a subsequent reinvestigation of the structure, we found the RBB molecule to be disordered. ${ }^{15}$ In addition, we have recently found a second phase of $\mathrm{RBB} / \mathrm{EG}$ at low temperatures $(93 \mathrm{~K}) .^{16}$

\section{EXPERIMENT}

\section{Materials and Crystal Growth}

RBB and ethyl 3,4,5-trihydroxybenzoate (EG) were purchased from Sigma-Aldrich and Wako Pure Chemical Industries, Ltd., respectively. Single crystals of the 1:1 complex of RBB with EG were grown by recrystallization from a toluene solution which includes an equimolar quantity of both chemicals. After $24 \mathrm{~h}$, a number of red crystals were obtained in the form of blocks.

\section{Equipment and Measurements}

Reflection data were collected on a R-axis rapid-F diffractometer from Rigaku using $\mathrm{Cu} K \alpha$ radiation $(\lambda=1.5418 \AA)$ at $-180{ }^{\circ} \mathrm{C}$ and room temperature. The structure was solved by direct methods [SIR2004 (Ref. 17)] and refinement was carried out by the full-matrix least-squares method on $F^{2}$ [SHELXL-97 (Ref. 18)].

Solution spectra were recorded on a UV-2400PC spectrophotometer (Shimadzu). Reflection spectra on single crystals were measured by means of a UMSP80 microscopespectrophotometer (Carl Zeiss). An Epiplan Pol $(\times 8)$ objective was used together with a Nicol-type polarizer. Reflectivities were corrected relative to the reflection standard of silicon carbide.

Simultaneous measurements of both diffraction diagram and differential scanning calorimetry (DSC) were also carried out on RBB/EG powders under $\mathrm{N}_{2}$ with a Rigaku Ultima III x-ray diffraction (XRD)/DSC differential scanning calorimeter over the temperature range between 20 and $200^{\circ} \mathrm{C}$. The heating rate was $2^{\circ} \mathrm{C} / \mathrm{min}$.

\section{Molecular Orbital Calculations}

Semiempirical molecular orbital (MO) calculations were performed using a QUANTUM CACHE ver. 3.2 program package $^{19}$ which includes MOPAC ver. 94.10 and the ZINDO programs. Geometry was optimized for protonated RBB, using the AM1 Hamiltonian. Optical absorption bands were calculated with the ZINDO program for the optimized geometry as well as for the $\mathrm{x}$-ray structure using the $x, y$, and $z$ coordinate set.
Table I. Crystallographic parameters for low- and high-temperature phases of $\mathrm{RBB} / \mathrm{EG}$

\begin{tabular}{lcc} 
& $\begin{array}{c}\text { Low-temp. phase } \\
\left(-180^{\circ} \mathrm{C}\right)\end{array}$ & High-temp. phase (RT) \\
\hline Formula & $\mathrm{C}_{74} \mathrm{H}_{80} \mathrm{O}_{16} \mathrm{~N}_{4}$ & $\mathrm{C}_{37} \mathrm{H}_{40} \mathrm{O}_{8} \mathrm{~N}_{2}$ \\
Molecular weight & 1281.42 & 640.71 \\
Crystal system & Triclinic & Triclinic \\
Space group & $\mathrm{P}$-1 & $P$-1 \\
$Z$ & 2 & 2 \\
$a(\AA)$ & $11.3689(2)$ & $11.4721(3)$ \\
$b(\AA)$ & $16.3654(3)$ & $11.8036(3)$ \\
$c(\AA)$ & $17.6518(3)$ & $12.4816(3)$ \\
$\alpha\left(\left(^{\circ}\right)\right.$ & $94.1760(7)$ & $85.805(2)$ \\
$\beta\left({ }^{\circ}\right)$ & $96.1440(7)$ & $87.202(1)$ \\
$\left.\gamma^{\circ}\right)$ & $93.7790(7)$ & $81.973(1)$ \\
$V\left(\AA^{3}\right)$ & $3247.69(10)$ & $1667.84(7)$ \\
$D e n s i t y ~\left(\mathrm{~g} / \mathrm{cm}^{3}\right)$ & 1.310 & 1.276 \\
\hline
\end{tabular}

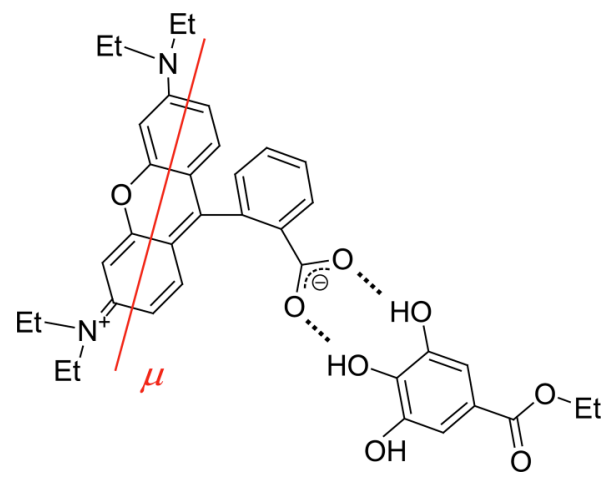

Figure 3. Schematic illustration of the zwitterionic structure of RBB/EG The direction of the transition moment $(\mu)$ as deduced from $M O$ calculations points along the long-molecular axis of the xanthene moiety.

\section{RESULTS AND DISCUSSION Crystal Structure}

Table I details the crystallographic parameters for the structure of $\mathrm{RBB} / \mathrm{EG}$ at $-180^{\circ} \mathrm{C}$ (Ref. 16) and at room temperature. ${ }^{15}$ The molecular conformation of the $1: 1 \mathrm{com}-$ plex of RBB/EG is schematically shown in Figure 3, illustrating that the lactone ring of RBB is opened to form a zwitterionic structure. There are two independent RBB/EG molecules in the unit cell in the low-temperature phase, as schematically shown in Figure 4(a) and designated RBB-A and RBB-B. In RBB-A, the ethyl groups of the diethylamino substituents at each extremity of the xanthene lie on the same side of the xanthene plane. On the other hand, in RBB-B, the ethyl groups of the diethylamino substituents lie on opposite sides of the xanthene plane. The dye/developer complexes (for example, RBB-A and EG-A) are formed through $\mathrm{OH} \cdots \mathrm{O}$ hydrogen bonds between the $\mathrm{O}$ atoms of the $\mathrm{RBB}$ molecule and the $\mathrm{OH}$ groups of the EG molecule. In addition, there are also $\mathrm{OH} \cdots \mathrm{O}$ intermolecular hydrogen bonds between two EG-A molecules. This forms 
(a)

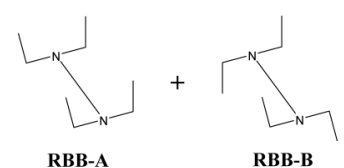

(b)

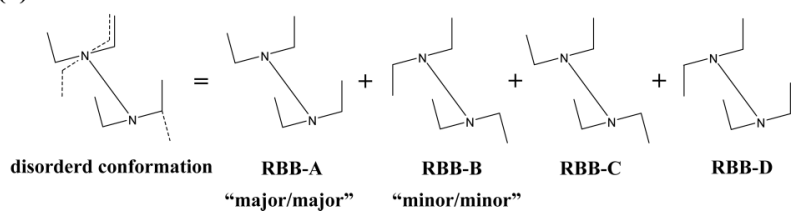

Figure 4. Schematic illustration of the molecular conformation: (a) low temperature phase and (b) RT phase. The ratio of the RBB-A/RBB-C in the room-temperature phase is about $7 / 3$

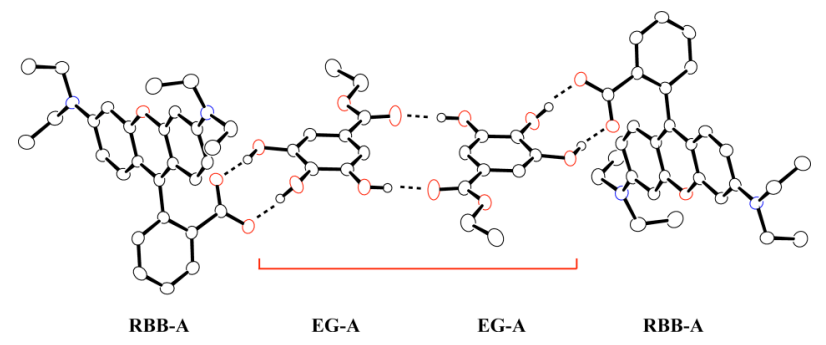

Figure 5. Dimerized structure of RBB/EG in the low-temperature phase: RBB-A $\cdots$ EG-A $\cdots$ EG-A $\cdots$ RBB-A.

a centrosymmetric hydrogen-bonded dimer (RBB-A $\cdots$ EG-A $\cdots$ EG-A $\cdots$ RBB-A) as shown in Figure 5. Likewise, another dimer structure is also possible in the lowtemperature phase (only RBB-B and EG-B). The dimerization of EG molecules alone in the middle of the complex is similar to that found in $n$-propyl gallate. ${ }^{20-22}$

The phenolic hydrogen atoms of EG as discussed in the Introduction are localized on Fourier density maps in both RBB-A and RBB-B. The O-H distances are 0.92(2)-0.94(4) $\AA$. This indicates that the proton transfer from EG to RBB is not the case in the solid state. Furthermore, in the carboxylate group $\left(-\mathrm{COO}^{-}\right)$, the $\mathrm{C}-\mathrm{O}$ distances are found to be nearly equal [bond lengths: about 1.254(2)-1.266(2) $\AA$ ]. This is due to the electron delocalization in the carboxylate as schematically shown in Fig. 3. Details are given in Ref. 16.

In the room-temperature phase, ${ }^{15}$ there is only one independent RBB/EG molecule in the unit cell. Furthermore, the RBB molecule is structurally disordered as shown in the left side of Fig. 4(b) with a methyl group of one ethyl substituent of a diethylamino group at one extremity of the xanthene moiety [occupancies: $0.735(5) / 0.265(5)$ ] as well as the entire diethylamino-substituent (i.e., the $\mathrm{N}$ atom and the associated $\mathrm{C}$ and $\mathrm{H}$ atoms) at the other end of the xanthene unit disordered over two positions [occupancies: 0.653(7)/ $0.347(7)]$. The present disordered structure can be separated into RBB-A ("major/major" combination), RBB-B ("minor/ minor"), along with RBB-C and RBB-D as shown on the right side of Fig. 4(b) on the basis of the low-temperature structure of RBB-A and RBB-B. Of these, the previous reported by Sekiguchi et al. ${ }^{10}$ only identified RBB-A

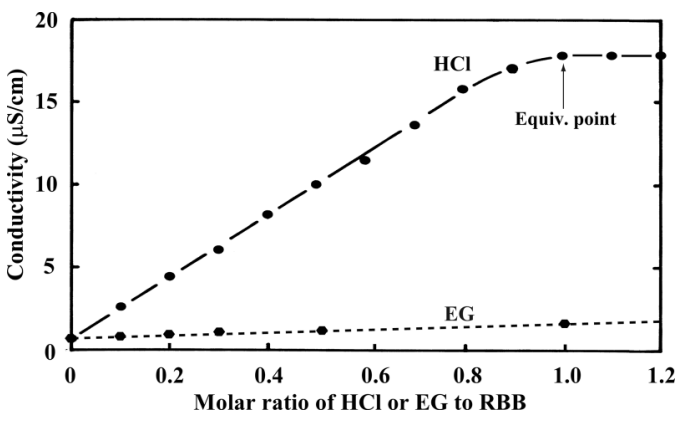

Figure 6. Conductometric titration of $\mathrm{RBB}$ with $\mathrm{HCl}$ or $\mathrm{EG}$ in a MEK solution.

(major/major). RBB and EG form a hydrogen-bonded dimer at room temperature, just as in the case of the lowtemperature phase. The existence of the four possible RBB conformations leads to the formation of 16 kinds of $\mathrm{RBB} \cdots \mathrm{EG} \cdots \mathrm{EG} \cdots \mathrm{RBB}$ dimers in the crystal.

\section{Color Generation of $\mathrm{RBB}$ in Solution with EG or $\mathrm{HCl}$}

The color generation of $\mathrm{RBB} / \mathrm{EG}$ is basically due to the acidbase reaction between EG (acid) and RBB (base). We monitored, therefore, simultaneously both the neutralization process and the resulting absorption spectra. The conductometric titration of $\mathrm{RBB}$ with $\mathrm{EG}$ or $\mathrm{HCl}$ provides us with valuable information about the percentage of the lactone ring opening.

Figure 6 shows the conductometric titration of $\mathrm{RBB}$ $\left(2 \times 10^{-4} \mathrm{M}\right)$ with $\mathrm{HCl}(0.01 \mathrm{M})$ or $\mathrm{EG}(0.01 \mathrm{M})$ in a methylethylketone (MEK) solution. With the $\mathrm{HCl}$ titration, the conductivity increases linearly and then becomes constant, showing the equivalence point at the molar ratio of $\mathrm{RBB}: \mathrm{HCl}=1: 1$. This indicates that the opening of the lactone ring is complete (i.e., 100\%). The absorption band at about $556 \mathrm{~nm}$ grows rapidly as shown in Figure 7(a) with the opening of the lactone ring accompanied by an isosbestic point around $340 \mathrm{~nm}$. The molar extinction coefficient for the complete ring opening is about 110,000 $\mathrm{L} \mathrm{mol}^{-1} \mathrm{~cm}^{-1}$. On the contrary, the titration with EG brings about no noticeable increase in electrical conductivity (Fig. 6). This indicates that EG is scarcely dissociated in MEK and that the lactone ring is still closed. In fact, the absorption band around $556 \mathrm{~nm}$ is negligibly small with the $1: 1$ ratio of $\mathrm{RBB}$ with EG, as shown in Fig. 7(b). The above results indicate that the protonation in solution (i.e., ring opening) occurs only with $\mathrm{HCl}$ not with EG.

\section{Color Generation of Spin-coated RBB by Protonation with Vapors of $\mathrm{HNO}_{3}$}

A similar protonation-experiment was also carried out in the solid state, using spin-coated RBB (thickness: about $230 \AA$ ). Protonation was carried out with vapor of $\mathrm{HNO}_{3}$ on spincoated RBB. The meaning of this experiment is to study the maximum available tinctorial strength induced by protons that can freely migrate throughout the spin-coated $\mathrm{RBB}$ to protonate all RBB molecules. Figure 8(a) shows the change in the absorption spectra as a function of exposed time. Coloration due to protonation with $\mathrm{HNO}_{3}$ proceeds quite 


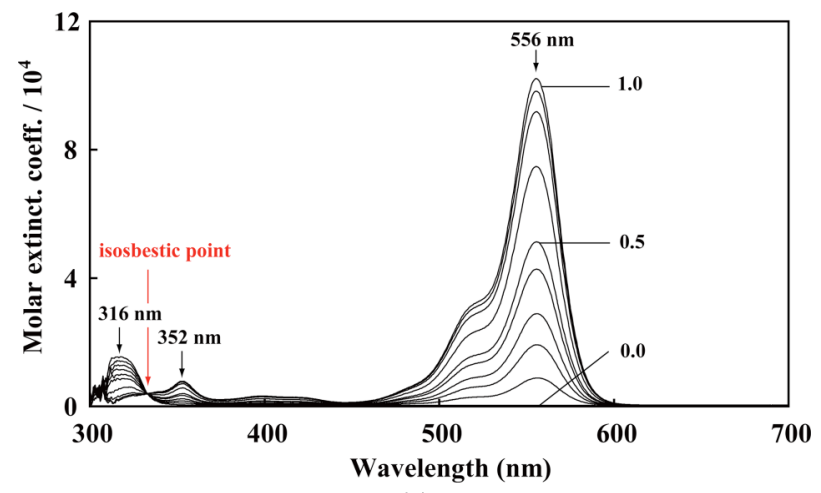

(a)

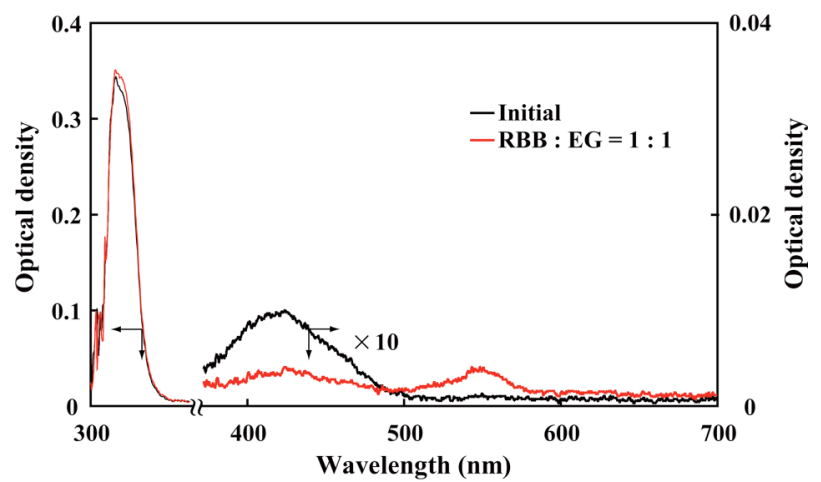

(b)

Figure 7. (a) Spectral change in RBB with $\mathrm{HCl}$ and (b) spectral change with EG.

rapidly and the saturation of the absorption spectrum (i.e., complete opening of the lactone ring) is observed in $2 \mathrm{~s}$. The complete opening is also borne out by the IR spectra [Fig. 8(b)], showing that the lactone ring at $1750 \mathrm{~cm}^{-1}$ disappears due to protonation. Instead, the four characteristic bands $\left(1615,1435,1368\right.$, and $\left.1270 \mathrm{~cm}^{-1}\right)$ for the carboxyl group appear new, accompanied by intensity enhancement of the $\mathrm{OH}$ stretching band around $3300 \mathrm{~cm}^{-1}$. The spectral change due to protonation is quite similar to that of the conductometric titration of $\mathrm{RBB}$ with $\mathrm{HCl}$ shown in Fig. 7(a). However, the absorption maximum occurs at 577 $\mathrm{nm}$ in spin-coated RBB while at $556 \mathrm{~nm}$ in solution, showing a bathochromic shifted by about $21 \mathrm{~nm}$ on going from solution to the solid state. The present shift can basically be interpreted in terms of the crystal shift upon crystallization. $^{11,12}$

\section{Spectral Shift of Spin-coated RBB/EG with Vapors of $\mathrm{HNO}_{3}$}

We then studied the influence of protonation with vapors of nitric acid on the spin-coated RBB/EG (=1:1) (thickness: about $300 \AA$ ) in order to study the percentage of the lactone ring opening. Figure 9(a) shows the absorption spectra of spin-coated RBB/EG and its additional vapor treatment with nitric acid. It should be noted that protonation with vapors of $\mathrm{HNO}_{3}$ displaces the absorption band to $577 \mathrm{~nm}$. Furthermore, vapor treatment remarkably enhances the tinctorial strength, indicating that there is a fraction of RBB molecules whose lactone ring is still closed in spin-coated RBB/EG. On

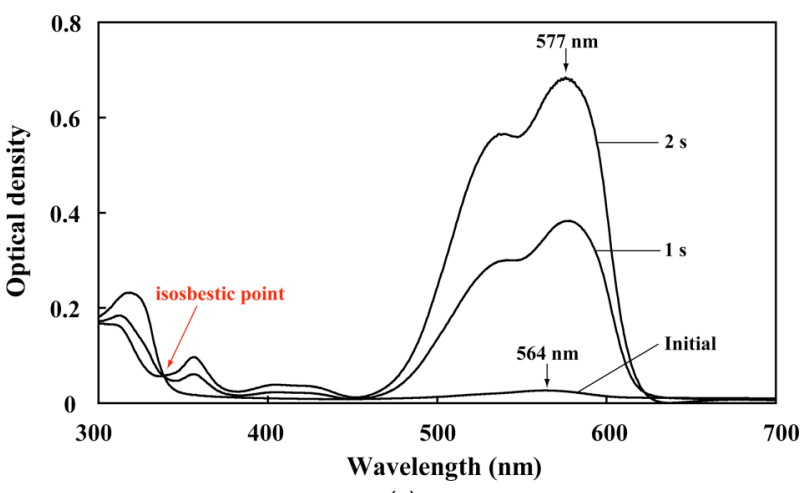

(a)

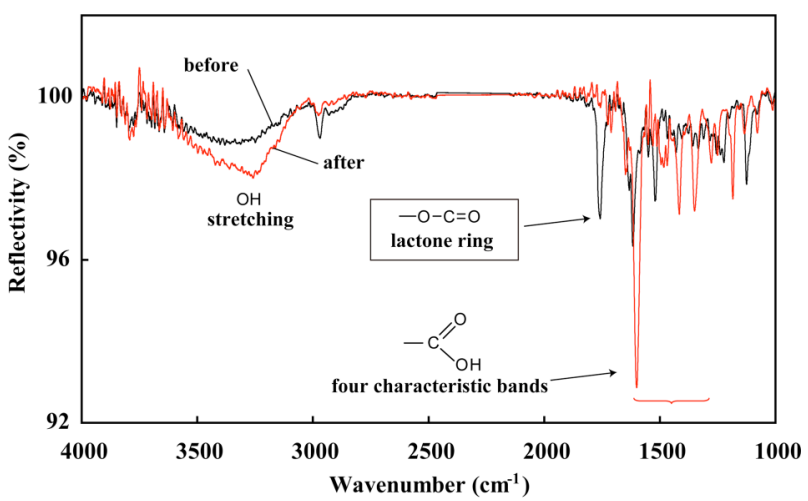

(b)

Figure 8. (a) Spectral change in the spin-coated RBB by protonation with vapors of $\mathrm{HNO}_{3}$ and (b) IR spectra before and after protonation. Film thickness: $230 \AA$.

the basis of the enhanced optical density, the percentage of the lactone ring opening prior to acid vapor treatment is estimated to be about $80 \%$. This signifies that the complete opening of the lactone ring is rather difficult in spin-coated films, presumably because of the steric hindrance of each of the component molecules. Similar or even greater steric hindrance will occur in practical systems composed of a mixture of leuco dye/developer particles. Fig. 9(b) is the XRD diagram for spin-coated RBB/EG, showing an amorphous phase. The halo diffraction pattern around $2 \theta=26^{\circ}$ is due to the glass substrate used.

Now, we discuss the difference in maximum absorption wavelength $\left(\lambda_{\max }\right)$ in the spin-coated RBB/EG $(567 \mathrm{~nm})$ and the spin-coated RBB protonated with $\mathrm{HNO}_{3}(577 \mathrm{~nm})$. We believe that the protonation (i.e., ring opening) of spincoated RBB by vapors of $\mathrm{HNO}_{3}$ proceeds quite easily and rapidly since the proton is a vanishingly small cation and thus can easily migrate to the bulk. In addition, the proton requires much less space for the opening of the lactone ring as compared with bulky EG. Therefore, protonation of spincoated RBB with vapors of $\mathrm{HNO}_{3}$ is expected to bring about a flatter xanthene plane than that of spin-coated RBB/EG. Table II shows the calculated absorption bands for the zwitterionic RBB molecule optimized by MO calculations and also for the $\mathrm{x}$-ray structure of the room-temperature phase. Our visual observation of both structures supports the inference that the geometry-optimized structure is entirely flat while slightly deformed in the $\mathrm{x}$-ray structure. As seen from 


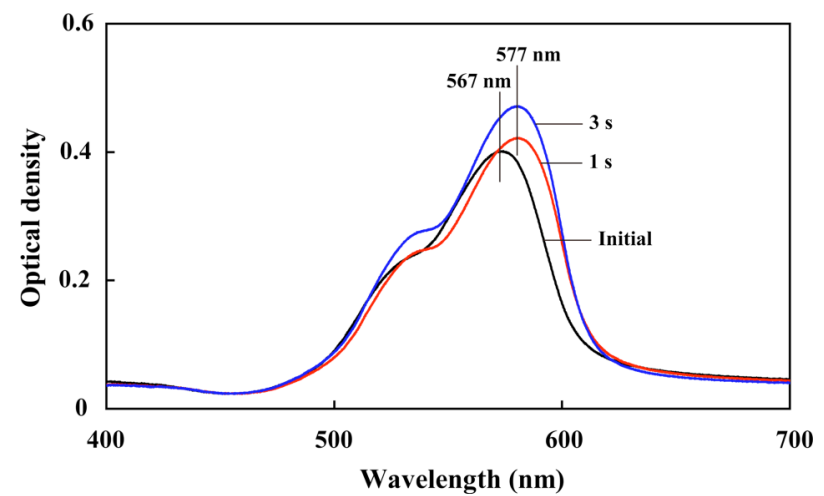

(a)

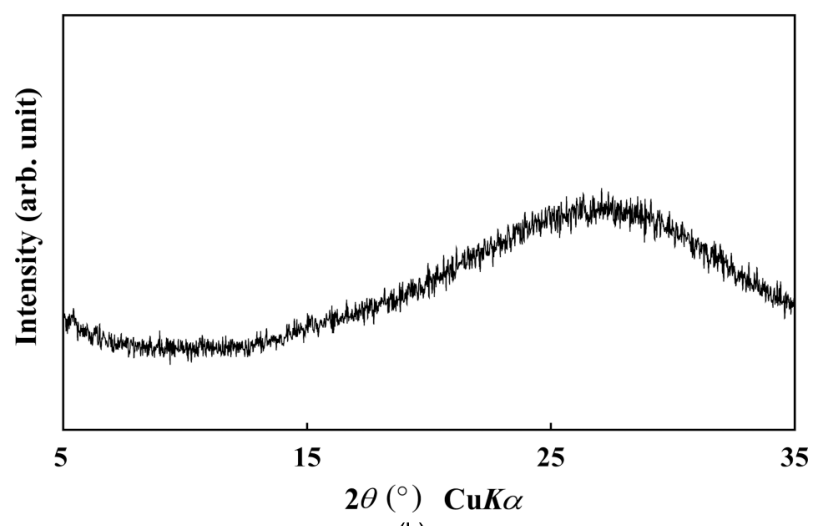

(b)

Figure 9. (a) Spectral change in the spin-coated RBB/EG by additional protonation with vapors of $\mathrm{HNO}_{3}$ (film thickness: $300 \AA$ ) and (b) XRD of spin-coated RBB/EG, showing an amorphous phase.

Table II. Optical absorption bands of RBB according to MO calculations.

\begin{tabular}{lcc} 
& \multicolumn{2}{c}{ Absorption band } \\
\cline { 2 - 3 } & $\begin{array}{c}\lambda \\
(\mathrm{nm})\end{array}$ & $f$ \\
\hline RBB $\left(\mathrm{H}^{+}\right)$Geo. Opt. & 455.8 & 1.13 \\
RBB $(\mathrm{x}$ ray) RT & 426.0 & 0.69 \\
\hline
\end{tabular}

Table II, the geometry-optimized structure gives an absorption band at longer wavelengths with enhanced oscillator strength than that of the $\mathrm{x}$-ray structure. This tendency is fairly in good agreement with the experimental result [Figs. $8(a)$ and $9(a)]$.

\section{Polarized Reflection Spectra Measured on Single Crystal of $R B B / E G$}

Polarized reflection spectra of $\mathrm{RBB} / \mathrm{EG}$ were measured on the (010) plane of single crystals by means of a microscopespectrophotometer. The direction of the transition moment as deduced from MO calculations points along the longmolecular axis of the zwitterionic structure as shown in Fig. 3. The molecular arrangement on the (010) plane of the crystal structure is shown in Figure 10. Figure 11 shows the polarized reflection spectra for polarization parallel or per-

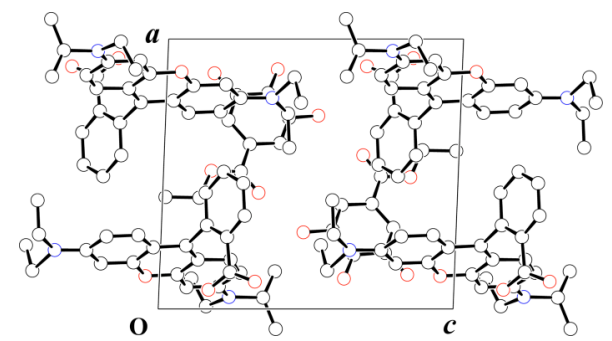

Figure 10. Molecular arrangement of RBB/EG molecules on the $(010)$ plane.

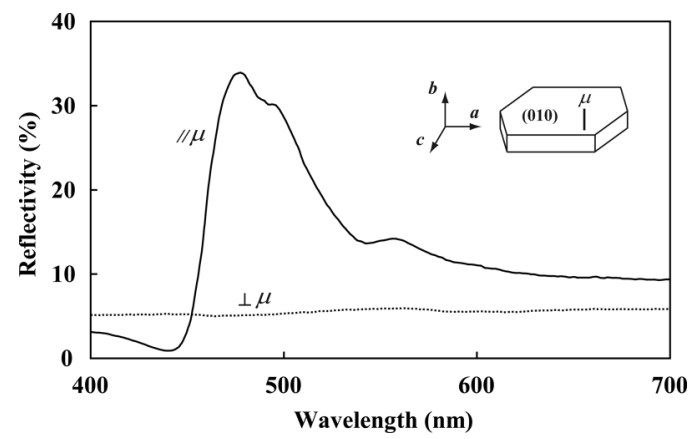

Figure 11. Polarized reflection spectra measured on the $(010)$ plane of $\mathrm{RBB} / \mathrm{EG}$ single crystals.

pendicular to the transition moment. A prominent reflection band appears around $480 \mathrm{~nm}$ for polarization parallel to the transition moment, together with two small peaks around 500 and $550 \mathrm{~nm}$. On the other hand, the reflection is totally quenched for polarization perpendicular to the transition moment. As judged from the reflection maximum around $480 \mathrm{~nm}$, the reflection band in single crystals is drastically displaced toward shorter wavelengths by about $100 \mathrm{~nm}$ as compared with that in spin-coated RBB/EG [Fig. 9(a)]. The present hypsochromic shift can basically be interpreted in terms of excitonic interactions described below.

In general, the interactions between excited molecules (i.e., so to speak, excitonic interactions) will appear to displace the absorption band toward longer or shorter wavelengths, depending on the molecular arrangement. ${ }^{11,12}$ This interaction is especially pronounced when the molar extinction coefficient is high as in the case of RBB/EG [over $10^{5}$ as shown in Fig. 7(a)] and the molecules are arranged periodically. According to the molecular exciton theory, ${ }^{11,12}$ the interaction energy $\left(\Delta E_{\text {exciton }}\right)$ is given by the dipole-dipole equation,

$$
\Delta E_{\text {exciton }}=|\mu|^{2}\left(1-3 \cos ^{2} \theta\right) / r^{3},
$$

where $\mu$ denotes the transition dipole, $r$ is the distance between two central points of two dipoles, and $\theta$ is the angle between one dipole and the vector connecting the two point dipoles. This interaction, however, falls off as the inverse cube of distance, so the most of the interaction should come from the nearest neighbors. The energetic shift is then determined by the strength of the interneighbor coupling $\left(|\mu|^{2}\right)$ which directly depends on the absorption coefficient 
(a)

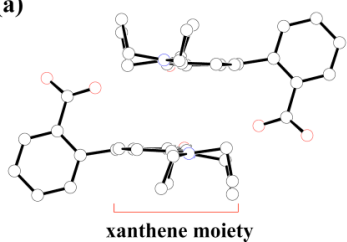

(b)

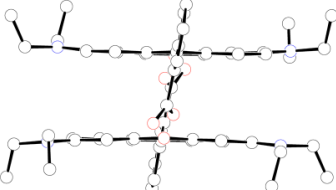

Figure 12. (a) Overlap of two of RBB molecules: (a) as seen from the short-molecular axis and (b) as seen from the long-molecular axis. The interplanar distance and the slip angle are about $3.51 \AA$ and $80^{\circ}$, respectively.

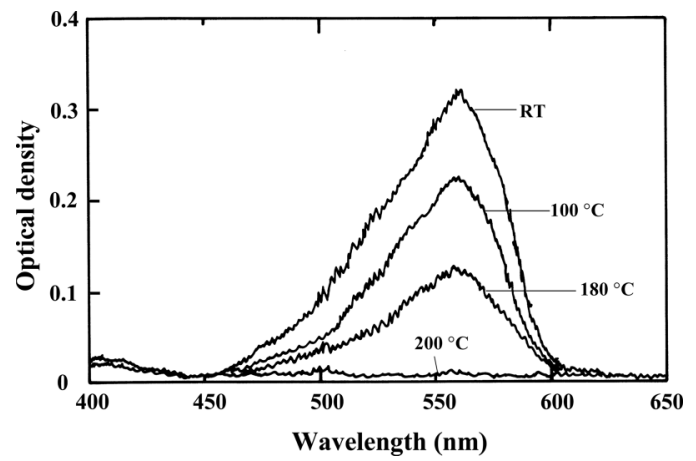

Figure 13. Absorption spectra of the spin-coated RBB/EG as a function of temperature. Film thickness: $220 \AA$

of the molecule, as well as the mutual relative orientation of the transition dipoles [i.e., $\left(1-3 \cos ^{2} \theta\right)$ ]. Below the critical angle of $\theta=54.7^{\circ}$, a J-aggregate-like bathochromic shift will result, and above it an $\mathrm{H}$-aggregate-like hypsochromic shift will be the case. Accordingly, a large hypsochromic shift occurs in the closely overlapped molecules shown in Figures 12 (a) and 12(b), as seen from the short and long-molecular axes, respectively. The interplanar distance and the slip angle are about $3.51 \AA$ and $80^{\circ}$, respectively. This suggests that strong excitonic interactions of the $\mathrm{H}$-aggregate type are operative in single crystals of $\mathrm{RBB} / \mathrm{EG}$ to displace the absorption band toward shorter wavelengths.

On the other hand, no excitonic interaction occurs in the amorphous phase as found in spin-coated RBB/EG [Fig. 9(b)]. Therefore, the absorption spectrum [(Fig. 8(a)] looks like the molecular spectrum in solution shown in Fig. 7(a).

\section{Appearance and Disappearance of the Color}

As discussed above, the appearance and disappearance of the color in spin-coated RBB/EG are due to the formation or collapse of $\mathrm{OH} \cdots \mathrm{O}$ hydrogen bonds between RBB and EG. Figure 13 shows the spectral change in spin-coated RBB/EG (thickness: about $220 \AA$ ) as a function of temperature. The discoloration begins at about $100^{\circ} \mathrm{C}$ and is completed around $200^{\circ} \mathrm{C}$. The discoloration is obviously correlated with the lactone ring closure that is induced by the weakening of $\mathrm{OH} \cdots \mathrm{O}$ hydrogen bonds due to the lattice vibration of the molecules at elevated temperatures.

Figure 14 shows the XRD and DSC curves, both of which were measured simultaneously. The diffraction diagram is shown on the left side, while the DSC curve is displayed on the right side. The diffraction peaks at room tem-

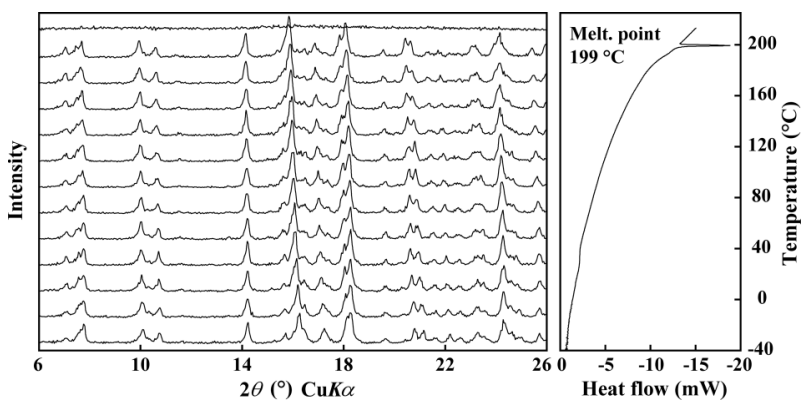

Figure 14. Simultaneous XRD/DSC diagrams as a function of temperature.

perature agree perfectly with the result of the structure analysis on single crystals. ${ }^{15}$ All diffraction peaks remain nearly intact in the temperature range between -40 and $225^{\circ} \mathrm{C}$, although the peaks around $2 \theta=16.3^{\circ}, 21.4^{\circ}$, and $25.8^{\circ}$ [(120), (221), and (3-10) planes, respectively] move slightly toward higher angles with increasing temperature. The present result indicates that no phase change occurs up to $199^{\circ} \mathrm{C}$, when the diffraction peaks disappear and powdered RBB/EG melts. As judged from the present result, the $\mathrm{RBB} / \mathrm{EG}$ complex is obviously not adequate for those rewritable imaging systems which are supposed to operate at relatively low temperature. It is interesting to note that practical rewritable leuco dye imaging systems are designed to comprise long chain developers that possess low melting points.

\section{CONCLUSIONS}

The electronic spectra of the 1:1 complex of RBB with EG have been investigated in solution and in the solid state on the basis of the crystal structure. The conclusions can be summarized as follows:

(1) There are two crystal phases in RBB/EG: low and room-temperature phases. In both phases, the RBB/EG complex forms a dimer $(\mathrm{RBB} \cdots \mathrm{EG} \cdots \mathrm{EG} \cdots \mathrm{RBB})$ through intermolecular $\mathrm{OH}^{*} \mathrm{O}$ hydrogen bonds.

(2) Protonation in solution occurs only with $\mathrm{HCl}$ not with EG.

(3) The percentage of the lactone ring opening is estimated to be about $80 \%$ in the spin-coated RBB/ EG.

(4) The difference in maximum absorption wavelength $\left(\lambda_{\max }\right)$ between the spin-coated RBB/EG and the spin-coated RBB protonated with vapors of $\mathrm{HNO}_{3}$ can be attributed to the planarity of the zwitterionic structure of the xanthene plane.

(5) The drastic difference in $\lambda_{\max }$ between the spincoated RBB/EG and the single crystals of RBB/EG is due to the difference in molecular arrangement. The former is characterized by randomly arranged molecules while the latter by periodically arranged molecules.

(6) Appearance and disappearance of the color in $\mathrm{RBB} / \mathrm{EG}$ corresponds to the formation or collapse of the hydrogen bond between RBB and EG, respectively. 


\section{ACKNOWLEDGMENTS}

The authors express sincere thanks to Rigaku Corporation for simultaneous measurements of XRD and DSC diagrams with a Rigaku Ultima III equipment.

\section{REFERENCES}

${ }^{1}$ R. Muthyala, Chemistry and Applications of Leuco Dyes (Plenum, New York, 1997).

${ }^{2}$ K. Tsutsui, T. Yamaguchi, and K. Sato, "Thermochromic properties of mixture systems of octadecylphosphonic acid and fluoran dye", Jpn. J. Appl. Phys. 33, 5925-5928 (1994).

${ }^{3}$ K. Naito, "Amorphous-crystal transition of organic dye assemblies: Application to rewritable color recording media”, Appl. Phys. Lett. 67, 211-213 (1995).

${ }^{4}$ S. M. Burkinshaw, J. Griffith, and A. D. Towns, "Reversibly thermolchromic systems based on ph-sensitive spirolactone-derived functional dyes", J. Mater. Chem. 13, 2677-2683 (1998).

${ }^{5} \mathrm{~J}$. Luthern and A. Peredes, "Determination of the stoichiometry of a thermochromic color complex via Job's method”, J. Mater. Sci. Lett. 19, 185-188 (2000).

${ }^{6}$ Y. Takahashi, A. Shirai, T. Segawa, T. Takahashi, and K. Sakakibara, "Why does a color-developing phenomenon occur on thermal paper comprising of a fluoran dye and a color developer molecule?", Bull. Chem. Soc. Jpn. 75, 2225-2231 (2002).

${ }^{7}$ J. Luthern and A. Peredes, "Determination of determination of the stoichiometry of a thermochromic complex via the method of continuous variation”, J. Mater. Sci. Lett. 22, 881-884 (2003).

${ }^{8}$ D. C. MacLaren and M. A. White, "Dye-developer interactions in the crystal violet lactone-lauryl gallate binary system: Implications for thermochromism”, J. Mater. Chem. 13, 1695-1700 (2003).

${ }^{9}$ D. C. MacLaren and M. A. White, "Competition between dye-developer and solvent-developer interactions in a reversible thermochromic system”, J. Mater. Chem. 13, 1701-1704 (2003).
${ }^{10}$ Y. Sekiguchi, S. Takayama, T. Gotanda, and K. Sano, "Molecular structures of the coloring species of a leuco dye with phenolic color developers", Chem. Lett. 36, 1010-1011 (2007).

${ }^{11}$ M. Kasha, Spectroscopy of the Excited State (Plenum Press, New York, 1976).

${ }^{12}$ D. P. Craig and S. H. Walmsley, Excitons in Molecular Crystals: Theory and Applications (W. A. Benjamin, Inc., New York, 1968).

${ }^{13}$ G. Rihs and C. D. Weis, "Interaction of color formers and coreactants. Part I. First crystal structures of the colorforming species composed of crystal violet lactone and metal iodides", Dyes Pigm. 15, 107-127 (1991).

${ }^{14} \mathrm{G}$. Rihs and C. D. Weis, "Interaction of color formers and coreactants. Part II. Crystal structure of a xanthene type colorformer and cadmium iodide", Dyes Pigm. 15, 165-173 (1991).

${ }^{15} \mathrm{~J}$. Mizuguchi and K. Sato, "A redetermination of 2-(6-diethylamino-3diethyliminio-3Hxanthen-9-yl)benzoate-ethyl gallate $(1 / 1)$ at room temperature", Acta Crystallogr. E65, o701-0702 (2009).

${ }^{16}$ J. Mizuguchi, "A low-temperature phase of the $1: 1$ complex of 2-(6-diethylamino-3-diethyliminio-3H-xanthene-9-yl)benzoate with ethyl gallate at 93 K”, Acta Crystallogr. E64, o1238-o1239 (2008).

${ }^{17}$ M. C. Burla, R. Caliandro, M. Camalli, B. Carrozzini, G. L. Cascarano, L. De Caro, C. Giacovazzo, G. Polidori, and P. Spagna, "SIR2004: An improved tool for crystal structure determination and refinement", J. Appl. Crystallogr. 38, 381-388 (2005).

${ }^{18}$ G. M. Sheldrick, "A short history of SHELX", Acta Crystallogr. 64, 112-122 (2008).

${ }^{19}$ QUANTUM CACHE, version 3.2 (Fujitsu, Ltd., Kanagawa, Japan) (1999).

${ }^{20} \mathrm{~S}$. Iwata, A. Hitachi, T. Makino, and J. Mizuguchi, " $n$-Propyl gallatechloroform (3/0.5)”, Acta Crystallogr. E61, o2587-o2589 (2005).

${ }^{21}$ A. Hitachi, T. Makino, S. Iwata, and J. Mizuguchi, " $n$-Propyl gallatedichloromethane (3/0.5)”, Acta Crystallogr. E61, o2590-o2592 (2005).

${ }^{22}$ J. Mizuguchi, A. Hitachi, S. Iwata, and T. Makino, " $n$-Propyl gallateacetonitrile (2/1)”, Acta Crystallogr. E61, o2593-o2595 (2005). 
(C)2009 Society for Imaging Science and Technology (IS\&T)

All rights reserved. This paper, or parts thereof, may not be reproduced in any form without the written permission of IS\&T, the sole copyright owner of The Journal of Imaging Science and Technology.

For information on reprints or reproduction contact

Donna Smith

Production Editor

The Journal of Imaging Science and Technology

Society for Imaging Science and Technology

7003 Kilworth Lane

Springfield, Virginia 22151 USA

703/642-9090 extension 107

703/642-9094 (fax)

dsmith@imaging.org

www.imaging.org 